\title{
Validación de la Versión Adaptada de la Escala de Auto-Ocultamiento de Larson \& Chastain en Universitarios Chilenos
}

\author{
Validation of the Adapted Version of the Larson \& Chastain Self-Concealment Scale \\ in Chilean University Students
}

\author{
Natalia Salinas-Oñate ${ }^{1}$, María José Baeza-Rivera² ${ }^{2}$ Belén Salinas-Rehbein ${ }^{3}$, Neli Escandón-Nagel ${ }^{4}$ y \\ Bertha Escobar-Alaniz ${ }^{5}$
}

\begin{abstract}
Resumen
El auto-ocultamiento se asocia a indicadores negativos de salud y a menores niveles de búsqueda de ayuda psicológica, sin embargo, en Chile no existen instrumentos que lo midan de manera confiable. En la presente investigación se validó la versión adaptada de la Escala de Auto-Ocultamiento de Larson \& Chastain en universitarios. Se tradujo y adaptó la escala a través de un enfoque por comité. Posteriormente, jueces expertos evaluaron su validez de contenido. Luego, se exploraron sus propiedades psicométricas en una muestra de 350 universitarios de La Araucanía, Chile. Se confirmó su estructura unifactorial, compuesta de ocho ítems, que evidencia invarianza escalar parcial entre hombres y mujeres, una buena consistencia interna $(\omega=.85)$, y que converge significativamente y en la dirección esperada con síntomas de depresión, ansiedad y estrés psicológico, e intención de buscar ayuda psicológica. Este instrumento representa un aporte en la detección de grupos de riesgo que con menor probabilidad buscarán ayuda para problemas de salud mental.
\end{abstract}

Palabras clave: auto-ocultamiento, búsqueda de ayuda psicológica, salud mental, validación, universitarios

\begin{abstract}
Self-concealment is associated with negative health indicators and lower levels of seeking psychological help, however, in Chile there are no scales that measure this construct reliably. In the present study the adapted version of Self-Concealment Scale was validated in university students. The scale was translated and adapted through a committee approach. Subsequently, expert judges evaluated its content validity. Then, the psychometric properties of the scale were evaluated in a sample of 350 university students from La Araucanía region, Chile. A unidimensional structure was confirmed, consisting of eight items, which shows partial scalar invariance between men and women, good internal consistency $(\omega=.85)$, and that converged in the expected direction and significantly with symptoms of depression, anxiety and stress, and intention to seek psychological help. This instrument represents a contribution in the detection of risk groups that are less likely to seek help for mental health problems.
\end{abstract}

Keywords: self-concealment, psychological help seeking, mental health, validation, university student

\footnotetext{
1 Doctora en Psicología. Profesora Asistente. Universidad de La Frontera. Avda. Francisco Salazar 01145, Temuco, Chile. Tel.: +56452325616. Correo: natalia.salinas@ufrontera.cl (Autora de correspondencia)

${ }^{2}$ Doctora en Psicología. Profesora Asistente. Universidad Católica de Temuco. Manuel Montt 56, Temuco, Chile. Tel.: +56452685079. Correo: maria.baeza@uct.cl

${ }^{3}$ Doctoranda en Psicología, Licenciada en Psicología. Estudiante de Doctorado en Psicología. Universidad de La Frontera. Avda. Francisco Salazar 01145, Temuco, Chile. Tel.: +56452325620. Correo: m.salinas03@ufromail.cl

${ }^{4}$ Doctora en Psicología Clínica y de la Salud. Profesora Instructora. Universidad Católica de Temuco. Manuel Montt 56, Temuco, Chile. Tel.: +56452685078. Correo: nescandon@uct.cl

${ }^{5}$ Magister en Salud Pública. Profesora Asistente. Universidad Católica de Temuco. Manuel Montt 56, Temuco, Chile. Tel.: +56452553860. Correo: bescobar@uct.cl
}

Revista Iberoamericana de Diagnóstico y Evaluación - e Avaliação Psicológica. RIDEP · No62 · Vol.1 · 51-65 · 2022 ISSN: 1135-3848 print /2183-6051online 


\section{Introducción}

Uno de los temas centrales en psicoterapia y psicología de la personalidad ha sido la tendencia a ocultar, deliberadamente, información personal a otros, denominado como auto-ocultamiento (Kelly \& Yip, 2006). El auto-ocultamiento se define como una predisposición a ocultar a otros, información personal que es percibida como estresante o negativa (Masuda et al., 2017), o es relativa a experiencias dolorosas y traumáticas (Larson \& Chastain, 1990). El auto-ocultamiento ha sido considerado como un rasgo de personalidad, que tendría dos procesos psicológicos a la base: la mantención de la privacidad a través del establecimiento de límites, y la gestión de la propia imagen (Larson \& Chastain, 1990). Otros autores (Akdoğan \& Çimşir, 2019) han planteado que existen otras disposiciones psicológicas que caracterizan a las personas que tienden a auto-ocultar información personal, como sentimientos de inferioridad, los cuales generan dificultad en los individuos para creer que son merecedores de amor y apoyo por parte de otros.

Pese a los lineamientos generales antes mencionados, coexisten distintas conceptualizaciones sobre este constructo. Para algunos autores (Kelly \& Yip, 2006), el autoocultamiento puede ser entendido como una predisposición biológica, que hace más vulnerable a aquellos individuos que la poseen a desarrollar síntomas de psicopatología. Masuda et al. (2011) han planteado que es un proceso de regulación del comportamiento, o un estado de ánimo desadaptativo, que podría promover, y aumentar, el malestar psicológico y físico.

El acto de auto-ocultar información relevante a otros ha demostrado tener un impacto negativo en desenlaces en salud tanto física como mental, relacionándose con ideación suicida (Friedlander et al., 2012), trastornos alimentarios (Masuda et al., 2011), dolores musculares y de cabeza, niveles más bajos de autoestima (Almas et al., 2011), y mayor sintomatología ansiosa y depresiva (Kahn \& Hessling, 2001).

Respecto a esto último, pareciera existir una relación bidireccional entre estas variables, donde el ocultar información dolorosa y traumática podría aumentar los niveles de malestar, elevando los síntomas ansiosos y depresivos (Larson \& Chastain, 1990), y a su vez, altos niveles de sintomatología depresiva y ansiosa tenderían a no ser develados a otros, y en consecuencia, tenderían a ocultarse (Kahn \& Garrison, 2009). Probablemente, esto se vincule a la vivencia de malestar, vergüienza y estrés asociado a la experiencia de auto-revelación (Vogel \& Wester, 2003).

Por otra parte, la evidencia internacional señala que quienes auto-ocultan información tienen más probabilidades de buscar ayuda profesional debido a que experimentan una mayor angustia. Sin embargo, el auto-ocultamiento también aumenta las actitudes negativas hacia la atención psicológica que inhibe el comportamiento de búsqueda de ayuda (Larson et al., 2015). Es esta última relación la que ha recibido una evidencia más robusta, señalándose que, quienes presentan mayores niveles de autoocultamiento son tres veces menos propensos a buscar ayuda profesional (Cepeda-Benito \& Short, 1998).

El auto-ocultamiento ha sido estudiado mayoritariamente en población universitaria (Masuda et al., 2010; Yukawa et al., 2007), evidenciándose que en muestras latinas (Mendoza et al., 2018) las mujeres presentan significativamente mayores niveles de autoocultamiento que los hombres. Dado que esta relación no se ha evidenciado en muestras de universitarios de otras etnias [ej. africanoamericanos (Masuda et al., 2012), asiáticoamericanos (Masuda et al., 2021), europeoamericanos (Masuda \& Boone, 2011)], se ha hipotetizado que estaría asociada al valor cultural denominado marianismo, referido a la idealización del rol de género femenino en latinoamericanas, donde se espera que la mujer sea: humilde, espiritualmente superior pero sumisa ante el hombre, capaz de hacer sacrificios y soportar sufrimientos extremos con dignidad y abnegación por el bien de la familia, lo que incluye guardar silencio para preservar la armonía social (Castillo \& Cano, 2007). Estas expectativas percibidas podrían conducir a un mayor ocultamiento de sí mismas (Castillo et al., 2010).

El focalizar el estudio del auto-ocultamiento en población universitaria ha estado vinculado a las tasas preocupantes de problemas de salud 
mental que se evidencian en este grupo. A nivel internacional, se señala que el 35\% reporta haber sufrido un trastorno mental, siendo el trastorno depresivo mayor el más común, con una prevalencia de $21.2 \%$ (Auerbach et al., 2018). Por otra parte, la etapa universitaria coincide con la denominada adultez emergente, caracterizada como una etapa de grandes contradicciones y de sentirse "en el medio" entre adolescencia y adultez (Arnett, 2015), acompañada de grandes cambios en el individuo, su contexto y sus roles, lo cual puede contribuir a la aparición de alteraciones de salud mental (Schulenberg et al., 2004). Además, se ha evidenciado que los estilos de vida de los estudiantes universitarios condicionan un contexto de riesgo para desarrollar problemas de salud mental (Aceijas et al., 2017).

En efecto, en la población universitaria chilena se han observado altas tasas de sintomatología, tal como se ha evidenciado en dos estudios locales, el primero de ellos reportó que un $28 \%$ de los estudiantes presentaba sintomatología depresiva (ELSOC-COES, 2018), mientras que el segundo señala que un $43 \%$ de los universitarios presenta sintomatología depresiva, un $47 \%$ tiene sintomatología ansiosa, un 53\% sintomatología de estrés, y un $28 \%$ de ellos presentaría de forma conjunta los tres tipos de síntomas (Barrera-Herrera et al., 2019). Por otra parte, se ha observado que un $5 \%$ presenta algún nivel de riesgo de cometer suicidio o autolesiones (Gómez et al., 2020), y un $24 \%$ presenta un consumo riesgoso de alcohol (Baader et al., 2014). De aquellos estudiantes que acuden a un servicio de salud estudiantil, se ha identificado que un $48 \%$ presenta trastornos adaptativos, un $22 \%$ muestra algún trastorno del ánimo, y un $21 \%$ tiene trastornos de ansiedad (Micin \& Bagladi, 2011).

Dada la relevancia de este constructo y las consecuencias negativas asociadas a éste, es importante contar con instrumentos válidos que permitan su medición apropiada, a fin de mejorar la detección de grupos en eventual riesgo de desarrollar las problemáticas de salud mental y física antes mencionadas.

Uno de los instrumentos más utilizados para su medición es la Escala de Auto-Ocultamiento o SCS por sus siglas en inglés (Self-Concealment Scale) de Larson y Chastain (1990). Esta escala consta de 10 ítems que miden tres aspectos del auto-ocultamiento: (1) tendencia a mantener las cosas para uno mismo, (2) poseer secretos angustiantes o pensamientos negativos sobre sí mismo, que han sido compartidos con pocas personas, y (3) aprehensión de revelar información personal. La SCS tiene un formato de respuesta tipo Likert, con 5 opciones de respuestas que van desde 1 (Muy en desacuerdo) hasta 5 (Muy de acuerdo), siendo un ejemplo de ítem: "Tengo pensamientos negativos sobre mi mismo que nunca comparto con nadie" (ítem original: $I$ have negative thoughts about myself that I never share with anyone). En el estudio original con una muestra de adultos, la escala evidenció una estructura unidimensional, y una alta consistencia interna $(\alpha=.83)$ (Larson \& Chastain, 1990).

La SCS ha sido validada en distintos contextos. Un meta-análisis llevado a cabo con 137 estudios realizados con poblaciones diversas (ej. distintas nacionalidades, estudios interculturales, población LGB, población adolescente, entre otros) señala que 99 de ellos reportaron coeficientes de consistencia interna adecuados, con una consistencia media general de $\alpha=.87$, y una estructura unifactorial (Larson et al., 2015). Asimismo, cuando esta escala se pone a prueba en población universitaria, se confirma su estructura unidimensional y niveles de confiabilidad apropiados, a saber: estudiantes canadienses $\alpha=.87$ (Cramer \& Barry, 1999), estudiantes latinos residente en Estados Unidos $\alpha=.86$ (Mendoza et al., 2015), estudiantes asiáticos, africanos y latinos residentes en Estados Unidos $\alpha=.87$ (Constantine et al., 2004).

Considerando la asociación que el autoocultamiento ha mostrado con la experimentación de problemas de salud mental, y la necesidad de pesquisar de manera temprana los casos que estén en riesgo de desarrollarlos, se hace necesario disponer de instrumentos que cuenten con evidencia de validez para el contexto universitario local. Es por ello que el objetivo general del presente estudio es validar la versión adaptada de la SCS en una muestra de universitarios chilenos.

\section{Método}

\section{Participantes}

A través de un muestreo no probabilístico por conveniencia, de tres meses de duración, se contactó a 350 estudiantes universitarios de entre 
18 a 29 años provenientes de dos universidades de la Región de La Araucanía, Chile.

Se excluyó del estudio a estudiantes de la carrera de Psicología, pues podrían sesgar sus respuestas en consideración del conocimiento que manejan respecto del constructo de autoocultamiento, producto de su formación profesional. Además, se excluyó a participantes que tuviesen una condición limitante para contestar los instrumentos, como discapacidad visual, cognitiva o de otro tipo.

\section{Instrumentos}

Los participantes contestaron un cuadernillo que contenía los siguientes instrumentos:

Cuestionario sociodemográfico. Creado adhoc para el estudio, en el cual se miden variables como edad, sexo, procedencia (urbana/rural), pertenencia a la etnia mapuche, religión y nivel socioeconómico (NSE) de los participantes. Esta variable fue medida a través de la escala ESOMAR (Adimark, 2000), la cual estima un NSE que es el resultante del cruce de las variables nivel educativo y ocupación de quien aporte el principal ingreso al hogar. Adicionalmente, se incluyen preguntas sobre experiencias previas en tratamiento psicológico, la cantidad de ocasiones en las que se estuvo en tratamiento, y la calidad percibida de este tratamiento.

Versión adaptada de la Escala de autoocultamiento de Larson y Chastain (1990). Esta escala conceptualiza el auto-ocultamiento como una tendencia a mantener las cosas en privado, caracterizada por la posesión de pensamientos secretos o negativos personalmente angustiantes, que se han compartido con pocas personas o con nadie, y aprensión por la divulgación de información personal oculta. La versión final adaptada quedó compuesta por ocho ítems con formato de respuesta tipo Likert de cinco puntos ( $I=$ Muy en desacuerdo a $5=$ Muy de acuerdo) (ej. "Cuando me pasa algo malo tiendo a guardarlo para m厃'), donde a mayor puntaje mayor auto-ocultamiento (más detalles sobre el proceso de adaptación y validación de este instrumento serán abordados en la sección de resultados) (Apéndice A).

Escalas de Depresión, Ansiedad y Estrés (DASS-21) (Lovibond \& Lovibond, 1995)versión adaptada para estudiantes universitarios Chilenos (Antúnez \& Vinet, 2012). Evalúa la presencia de estados afectivos negativos de depresión, ansiedad y estrés. Consta 21 ítems de auto-reporte con formato de respuesta de escala tipo Likert de cuatro puntos $(0=$ No describe nada de lo que me pasó o sentí en la semana a $3=$ Sí, esto me pasó mucho, o casi siempre). En la presente muestra evidenció un excelente índice de confiabilidad para la escala total y por subescalas $\left(\omega_{\text {total }}=.95 ; \omega_{\text {depresion }}=.90\right.$; $\left.\omega_{\text {ansiedad }} .87 ; \omega_{\text {estrés }}=.88\right)$.

Cuestionario General de Búsqueda de Ayuda (GHSQ-V) (Wilson et al., \& Thomas, 2011) - versión adaptada de Olivari y GuzmánGonzález (2018). Evalúa la probabilidad de buscar ayuda de distintas fuentes para seis tipos de problemas de salud mental (estrés, ansiedad, depresión, ideación suicida, abuso de sustancias y psicosis) presentados a través de viñetas. El respondiente debe indicar la probabilidad de buscar ayuda en fuentes formales (ej. Psicólogo/a) e informales (ej. Amigos/as) a través de una escala tipo Likert de 5 puntos ( $1=$ Muy poco probable a $5=$ Muy probable). Las propiedades psicométricas de esta escala han sido probadas en estudiantes universitarios de la región de $\mathrm{La}$ Araucanía, Chile (Silva et al., 2022) exhibiendo una estructura bi-factorial y buenos niveles de confiabilidad ( $\alpha$ formal=.86; $\alpha$ informal=.70). Para el presente estudio sólo se consideró la fuente formal de ayuda psicológica $(\omega=.87)$.

\section{Procedimiento}

Previa autorización de las correspondientes autoridades de pregrado y directores de carrera de las universidades seleccionadas, los participantes fueron contactados en sus salas de clases por un equipo de investigación compuesto por la investigadora principal, un estudiante de magíster y dos estudiantes de pregrado debidamente entrenados para la toma de datos. Luego de explicarles los objetivos del estudio, las características del proceso y de enfatizar el carácter voluntario y confidencial de su participación, los participantes recibieron dos copias del consentimiento informado donde se explicitaron todos los detalles del estudio. Quienes decidieron participar firmaron el consentimiento y contestaron los instrumentos. Este procedimiento se enmarca en un proyecto de investigación que consideró la aplicación de otros 
instrumentos, lo que tomó aproximadamente 40 minutos. El tiempo que invirtieron en participar fue compensado con tres dólares. La toma de datos se realizó durante los meses de abril y junio del año 2019.

El protocolo de esta investigación fue aprobado por el Comité Ético Científico de la Universidad de La Frontera y, por tanto, cumple los principios éticos promulgados por la declaración de Helsinki.

\section{Análisis de datos}

A continuación, los análisis serán descritos considerando las siguientes etapas de investigación: (a) traducción y validación de contenido, y (b) evaluación de propiedades psicométricas.

I. Traducción y validación de contenido. Para esta primera etapa un comité de tres investigadores bilingües tradujo la SCS de manera independiente. Luego esta información fue triangulada en una reunión de comité. Posteriormente, esta versión de la escala en español, pasó por un proceso de prueba de su validez de contenido, en el cual nueve jueces expertos evaluaron la coherencia y suficiencia de los ítems para medir el constructo (los criterios de inclusión de los jueces fueron desempeñarse como psicólogos en el área clínica y/o tener experiencia en investigación). Posteriormente, las respuestas de los jueces expertos fueron ingresadas a una base de datos utilizando el Software SPSS versión 22. Para el análisis de sus respuestas se traspuso la base de datos (de modo que los jueces fuesen columnas y los ítems las filas) y se calculó la correlación ítem-test corregida de cada ítem con la finalidad de evaluar la presencia de jueces que divergen de las evaluaciones del resto (teniendo correlaciones cercanas a cero o negativas). Posteriormente, se calculó el coeficiente de correlación intra-clase usando el modelo de efectos aleatorios con acuerdo absoluto. Para su interpretación se utilizaron los criterios propuestos por Koo y Li (2016), donde coeficientes menores a .5 se consideran pobres, entre .5 y $\quad .75$ moderados, entre .76 y .90 buenos, y mayores a .90 se consideran excelentes.

Posteriormente, se estimó el grado de concordancia de las puntuaciones dadas, a través del coeficiente V de Aiken (Aiken, 1980; 1985), cuyos valores fluctúan de 0 a 1 . Se aceptaron ítems cuyo valor fue mayor o igual a .80 , se evaluaron de manera especial aquellos ítems cuyo valor fue entre .70 y .79 y se rechazaron aquellos menores a .70, considerando además las sugerencias de los jueces expertos respecto modificaciones en la redacción de los ítems.

Finalmente, y con el objetivo de asegurar la comprensión de los ítems, se realizó un pilotaje con 10 estudiantes universitarios, con una entrevista cognitiva (técnica think-aloud), donde los sujetos retroalimentaron respecto de su impresión de los ítems y las posibles contradicciones que les podría generar cada alternativa. A partir de este proceso, no se detectaron dificultades específicas en el proceso de respuesta al instrumento.

\section{Evaluación de propiedades} psicométricas. Para esta segunda etapa, se trabajó con una matriz de datos en el software STATA 14.0. Inicialmente se realizó un tratamiento preliminar de los datos (limpieza de datos, imputación, análisis de casos atípicos multivariados). Posteriormente, se realizaron pruebas descriptivas para caracterizar la muestra.

Luego, para confirmar la estructura unidimensional de la escala, evidenciada por estudios anteriores (ej. Masuda et al., 2010; Mendoza et al., 2015; Yukawa et al., 2007) se procedió a realizar un Análisis Factorial Confirmatorio (AFC). Debido a que el supuesto de normalidad multivariada no fue asumido, la estimación de los modelos fue realizada con la corrección de Satorra-Bentler, incluyendo SatorraBentler chi-cuadrado $\left(\mathrm{SBx}^{2}\right)$, el Índice de ajuste comparativo (CFI>.95), Índice de Tucker Lewis (TLI>.90), el error cuadrático Medio de aproximación (RMSEA $\leq .06)$ y la Media Estandarizada de la Raíz Cuadrática Residual (SRMR<.05) (Ortiz \& Fernández-Pera, 2018). Adicionalmente, el test de Lagrange Multiplier fue ejecutado de acuerdo a consideraciones teóricas y empíricas.

Posteriormente, para determinar si el instrumento fue invariante entre hombres y mujeres se realizó un análisis de ecuaciones estructurales de multigrupo, siguiendo las recomendaciones de Byrne (2008). En este proceso, primero se estimó la invarianza configural, posteriormente la invarianza métrica y 
finalmente la invarianza escalar. Los modelos fueron contrastados por la diferencia del valor absoluto de chi-cuadrado y los grados de libertad (Likelihood Ratio Test), utilizando los valores obtenidos del modelo de invarianza configural como valores de referencia.

Luego, para estimar la confiabilidad de los factores se usó el estadístico omega de McDonald (McDonald, 1999). Para finalizar, se realizaron análisis de correlaciones bivariadas de Pearson para estimar la validez convergente de la escala con los constructos de depresión, ansiedad y búsqueda de ayuda psicológica.

Para todos los análisis antes descritos se consideró un alfa nominal de .05.

\section{Resultados}

A continuación, se reportan los resultados considerando los siguientes apartados: (a) validez de contenido, y (b) evaluación de propiedades psicométricas.

\section{Validez de contenido}

Respecto a la evaluación de los jueces expertos, en primer lugar, se consideraron las respuestas de ocho jueces (la evaluación de un juez fue desestimada, luego de evaluar las respuestas divergentes), de las cuales se obtuvo un coeficiente de correlación intra-clase moderado y estadísticamente significativo (ICC=.71, $p=.003$ ), lo que indica acuerdo inter-jueces. A partir de estos insumos, y considerando el coeficiente $\mathrm{V}$ de Aiken de cada ítem, se eliminaron dos reactivos por presentar valores menores a .70. Los reactivos eliminados fueron el 4 ("algunos de mis temas personales realmente me han atormentado") y el 6 ("a menudo tengo miedo de contar algo que no quiero"). En el caso del ítem número 4, los jueces expertos evalúan que el reactivo no mide directamente el acto de ocultar la información, sino la cualidad "tormentosa" de los problemas percibidos por las personas. Respecto del ítem 6 , señalan que no se enfatiza que el tema pertenezca a la esfera personal (ej. podría aludir a un rumor).

Finalmente, se acogieron los comentarios hechos por los jueces expertos, respecto de la modificación del lenguaje de algunos reactivos. Particularmente, se reemplazó la palabra "secreto" por "temas o asuntos personales", dado que - en el contexto cultural local- la primera acepción alude de manera casi exclusiva a problemas que generan montos elevados de angustia, generalmente asociados a temáticas familiares que no deben ser develadas. Por el contrario, "temas o asuntos personales" es una acepción que, si bien hace referencia a temáticas del mundo privado, es más inclusiva de otros asuntos estresantes o problemáticos para la persona.

\section{Propiedades psicométricas}

La muestra final, sobre la cual se probaron las propiedades psicométricas de la escala, quedó compuesta por 339 sujetos (11 sujetos fueron eliminados tras el análisis de casos atípicos). Los participantes tuvieron un promedio de edad de 20.2 años ( $D E=1.5$ años). Respecto del sexo la muestra estuvo balanceada, donde hay un porcentaje levemente mayor de mujeres (55.5\%), predominantemente de procedencia urbana (83.3\%), no Mapuches (73.6\%), pertenecientes a niveles socioeconómicos medios $(18.5 \%$ medio bajo, $26.5 \%$ medio, $35.3 \%$ medio alto). Durante el año académico, la mayor parte de los participantes (55.4\%) residen en Temuco sin su familia nuclear, $\mathrm{y}$ un porcentaje minoritario (21.3\%) trabaja además de estudiar.

Respecto de los antecedentes clínicos de la muestra, se observa que un $42.6 \%$ presenta sintomatología ansiosa, $42.6 \%$ depresiva y $52.9 \%$ de estrés psicológico. Además, un porcentaje minoritario (5.9\%) se encuentra consumiendo fármacos para el manejo de síntomas ansiosos y/o depresivos, un porcentaje similar (4.7\%) se encuentra en tratamiento psicológico en la actualidad y un $40.1 \%$ estuvo en tratamiento psicológico anteriormente. De estos, la mayoría califican esa experiencia previa como de "poca" (34\%) o "regular" (34\%) utilidad (ver más detalles en Tabla 1).

Respecto del AFC, inicialmente se probó una solución inicial unifactorial. Se comparó este modelo (modelo 1) con uno alternativo (modelo 2) en donde se agregaron covarianzas entre los errores, considerando la sugerencia del Test de Lagrange Multiplier y criterios teóricos que se discutirán posteriormente. Los indicadores de bondad de ajuste para ambos modelos se presentan en la Tabla 2. 
Tabla 1. Características sociodemográficas de la muestra $(\mathrm{n}=339)$

\begin{tabular}{|c|c|}
\hline Variables & Descriptivos \\
\hline Edad $(M)$ & $20.2(D E=1.5)$ \\
\hline \multicolumn{2}{|l|}{$\operatorname{Sexo}(\%)$} \\
\hline Hombre & 44.5 \\
\hline Mujer & 55.5 \\
\hline \multicolumn{2}{|l|}{ Procedencia $(\%)$} \\
\hline Urbana & 83.3 \\
\hline Rural & 16.7 \\
\hline \multicolumn{2}{|l|}{ Pertenencia a etnia mapuche $(\%)$} \\
\hline $\mathrm{Si}$ & 26.4 \\
\hline No & 73.6 \\
\hline \multicolumn{2}{|l|}{ Nivel socioeconómico (\%) } \\
\hline Bajo & 5.6 \\
\hline Medio bajo & 18.5 \\
\hline Medio & 26.5 \\
\hline Medio alto & 35.3 \\
\hline Alto & 8.8 \\
\hline Muy alto & 4.4 \\
\hline \multicolumn{2}{|l|}{ Residencia durante el año académico (\%) } \\
\hline Con familia nuclear & 44.6 \\
\hline Sin familia nuclear (pensión, arriendo, otro) & 55.4 \\
\hline \multicolumn{2}{|l|}{ Ocupación (\%) } \\
\hline Sólo estudiar & 78.8 \\
\hline Estudio y trabajo part-time & 20.0 \\
\hline Estudiar y trabajar jornada completa & 1.3 \\
\hline \multicolumn{2}{|l|}{ Síntomas de ansiedad $(\%)$} \\
\hline $\mathrm{Si}$ & 42.6 \\
\hline No & 57.4 \\
\hline \multicolumn{2}{|l|}{ Síntomas depresivos (\%) } \\
\hline $\mathrm{Si}$ & 42.6 \\
\hline No & 57.4 \\
\hline \multicolumn{2}{|l|}{ Síntomas de estrés psicológico (\%) } \\
\hline $\mathrm{Si}$ & 52.9 \\
\hline No & 47.1 \\
\hline \multicolumn{2}{|c|}{ Consumo de fármacos para síntomas ansiosos y/o depresivos (\%) } \\
\hline $\mathrm{Si}$ & 5.9 \\
\hline No & 94.1 \\
\hline \multicolumn{2}{|l|}{ Tratamiento psicológico actual (\%) } \\
\hline $\mathrm{Si}$ & 4.7 \\
\hline No & 95.3 \\
\hline \multicolumn{2}{|l|}{ Tratamiento psicológico anterior (\%) } \\
\hline $\mathrm{Si}$ & 40.1 \\
\hline No & 59.9 \\
\hline \multicolumn{2}{|c|}{ Utilidad percibida de tratamiento psicológico anterior (\%) } \\
\hline Nada & 14 \\
\hline Poca & 34 \\
\hline Regular & 34 \\
\hline Bastante & 9 \\
\hline Mucho & 9 \\
\hline
\end{tabular}

Cuando se compara el modelo 1 y 2 se observa una diferencia estadísticamente significativa. Además, el modelo 2 exhibe mejores indicadores de bondad de ajuste que el modelo 1 . Dadas estas diferencias, junto con la relevancia teórica del segundo modelo, este último fue escogido.

Tal como se aprecia en la Figura 1, los ocho ítems cargan en una única dimensión, y todos poseen cargas factoriales superiores a .30 (la más baja fue de .45).

El test de Langrange Multiplier sugirió correlacionar algunos errores, específicamente entre los ítems: (a) 2 ("Si compartiera todos mis temas personales con mis amigos, les agradaría menos") у 7 ("Mis asuntos personales son demasiado vergonzosos como para compartirlos"), donde se observan semejanzas de contenido, aludiendo ambos a la dificultad de compartir asuntos personales con otros; (b) 3 ("Hay muchas cosas sobre mí que me guardo") y 4 ("Cuando me pasa algo malo, tiendo a guardármelo"), donde ambos ítems apuntan al acto de guardar información para sí mismo/a; y (c) 4 (anteriormente mencionado) y 8 ("Tengo pensamientos negativos sobre mí mismo que nunca comparto con nadie"), donde ambos ítems aluden a la vivencia de experiencias calificadas negativamente y que son reservadas en la esfera personal. Con todo esto, esta estructura unidimensional resultó teóricamente plausible. 
Tabla 2. Indicadores de bondad de ajuste de los modelos testeados

\begin{tabular}{|c|c|c|c|c|c|c|c|}
\hline Modelos & $\chi^{2}(g l)$ & $\chi 2 / g l$ & CFI & TLI & RMSEA (IC-90\%) & SMRS & $\Delta \chi^{2}(\Delta \mathrm{gl})$ \\
\hline Modelo 1 & $\begin{array}{c}127,91 \\
(20)\end{array}$ & 6,40 & .88 & .83 & $\begin{array}{c}.13 \\
(.11-.15)\end{array}$ & .06 & -- \\
\hline Modelo 2 & $\begin{array}{c}52,61 \\
(17)\end{array}$ & 3,09 & .96 & .94 & $\begin{array}{c}.08 \\
(.06-.11)\end{array}$ & .04 & $\begin{array}{c}75,3^{*} \\
(3)\end{array}$ \\
\hline
\end{tabular}

Modelo 1=Un factor sin covarianza entre errores; Modelo 2=Un factor con tres covarianzas entre errores. $* p<.001$

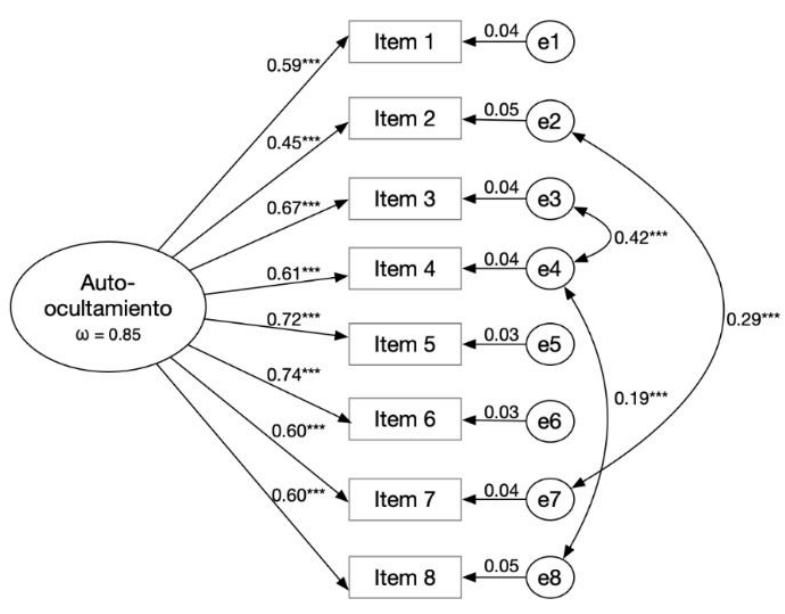

Figura 1. Análisis Factorial Confirmatorio de la

Escala de Auto-ocultamiento en estudiantes universitarios $(\mathrm{n}=339)$

Respecto del análisis de invarianza de la escala, inicialmente se probó el ajuste del modelo por separado para ambos grupos (hombres y mujeres), evidenciándose adecuados índices de bondad de ajuste para hombres $\left(\chi^{2} \quad{ }_{(17)}=24.96\right.$, $p<.001$, CFI $=.98$, TLI $=.97$, RMSEA $=.06$ [.00$.10]$, SRMR=.04) y mujeres $\left(\chi_{(17)}^{2}=50.60, p<.001\right.$, $\mathrm{CFI}=.94, \quad \mathrm{TLI}=.90, \quad \mathrm{RMSEA}=.10 \quad[.07-.14]$, $\mathrm{SRMR}=.05)$. Posteriormente, se siguieron los siguientes pasos:

Modelo de referencia. Este análisis se realizó con el sexo como variable de agrupación. Los resultados indican un excelente ajuste a los datos $\left(\chi^{2} \quad{ }_{(61)}=110.88, \quad p<.001, \quad\right.$ CFI $=.95, \quad$ TLI $=.95$, RMSEA=.07 [.05-.09], SRMR=.06).

Prueba de invarianza configural o misma forma. Este análisis requiere que -para ambos grupos- los ítems carguen en el mismo factor, por lo tanto, en este paso no se impuso ninguna restricción en las cargas, observándose un buen ajuste $\left(\chi^{2}{ }_{(34)}=75.56, p<.001, \mathrm{CFI}=.96\right.$, TLI $=.93$, RMSEA $=.09 \quad[.06-.01], \quad$ SRMR=.05). Estos indicadores se utilizaron para contrastar con los modelos posteriores.

Prueba de la invarianza métrica. En este paso se buscó probar si la escala tiene el mismo sentido semántico en ambas muestras comparadas, lo que implicó imponer una restricción de igualdad en las cargas factoriales de hombres y mujeres. Los indicadores de bondad de ajuste fueron excelentes $\left(\chi^{2} \quad{ }_{(41)}=88.59, \quad p<.001, \quad\right.$ CFI $=.95, \quad$ TLI $=.93$, RMSEA $=.08$ [.06-.11], SRMR=.07). Al realizar la comparación de este modelo más restrictivo con el menos restrictivo (configural), no se observan diferencias estadísticamente significativas $(\Delta \chi 2(7)$ 13.07, $\Delta \mathrm{p}=.07$ ), concluyéndose que el modelo de medida opera de la misma manera para hombres y mujeres, es decir, el significado de la escala de auto-ocultamiento es similar para ambos grupos.

Prueba de invarianza estructural o equivalencia escalar. Dado que en los pasos previos se demostró que el modelo teórico posee la misma forma y la escala tiene un significado similar en ambas muestras, en este paso se sumaron a las restricciones de igualdad, la restricción de los interceptos. Tal como se aprecia en la Tabla 3, los indicadores de bondad de ajuste son excelentes $\left(\chi^{2}\right.$ (49) $=104.36, \quad p<.001, \quad$ CFI $=.94, \quad$ TLI $=.93$, RMSEA=.08 [.06-.10], SRMR=.07). Al comparar este modelo con el modelo configural se obtuvo una diferencia estadísticamente significativa $\left(\Delta \chi^{2}{ }_{(15)}=28.80, \Delta p=.02\right)$, sugiriendo diferencias en al menos uno de los ítems entre hombres y mujeres.

Prueba post-estimación. Utilizando el test de Wald, se observaron diferencias estadísticamente significativas en los ítems $3\left(\chi_{(1)}^{2}=5.05, p=.025\right)$ y 4 $\left(\chi^{2}(1)=6.15, p=.013\right)$ entre hombres y mujeres, lo que sugiere que se logró una invarianza escalar parcial. Posterior a esto, se procedió a liberar las cargas de los interceptos de los ítems 3 y 4 , y se calcularon los indicadores de bondad de ajuste para este modelo, los cuales fueron excelentes $\left(\chi^{2}\right.$ ${ }_{(41)}=88.66, p<.001, \mathrm{CFI}=.95, \mathrm{TLI}=.93, \mathrm{RMSEA}=.08$ [.06-.10], SRMR=.07). Finalmente, el modelo de invarianza escalar con interceptos liberados fue comparado con el modelo configural, no observándose diferencias estadísticamente significativas entre ellos $\left(\Delta \chi^{2}(7)=13.10, \Delta p=.07\right)$, lo que indica que se logró invarianza escalar parcial. 
Tabla 3. Análisis de invarianza con sexo como variable de agrupación

\begin{tabular}{lcccccccccc}
\hline Modelo & $\chi^{2}$ & $g l$ & $C F I$ & $T L I$ & $R S M E A$ & $90 \%$ IC & SRMR & $\Delta \chi^{2}$ & $\Delta \mathrm{gl}$ & $\Delta p$ \\
\hline Modelo para hombres & 24.96 & 17 & .98 & .97 & .06 & {$[.00-.10]$} & .04 & -- & -- & -- \\
Modelo para mujeres & 50.60 & 17 & .94 & .90 & .10 & {$[.07-.14]$} & .05 & -- & -- & -- \\
Modelo sin & 110.88 & 61 & .95 & .95 & .07 & {$[.05-.09]$} & .06 & -- & -- & -- \\
restricciones & 75.56 & 34 & .96 & .93 & .09 & {$[.06-.11]$} & .05 & -- & -- & -- \\
Invarianza configural & 88.59 & 41 & .95 & .93 & .08 & {$[.06-.11]$} & .07 & 13.07 & 7 & .07 \\
Invarianza métrica & 104.36 & 49 & .94 & .93 & .08 & {$[.06-.10]$} & .07 & 28.80 & 15 & .02 \\
Invarianza escalar & 88.66 & 41 & .95 & .93 & .08 & {$[.06-.10]$} & .07 & 13.10 & 7 & .07 \\
Invarianza escalar & & & & & & .93 &
\end{tabular}

$\chi^{2=}$ chi cuadrado; $\mathrm{gl}=$ Grados de libertad; $\mathrm{CFI}=$ Índices de ajuste comparativo; TLI=Índice Tucker Lewis; RMSEA=Raíz cuadrada del error medio cuadrático; SRMR=Raiz Cuadrada Media de los Residuos Estandarizados

Respecto a la confiabilidad de la escala se obtuvo coeficiente Omega de McDonald de .85 .

En cuanto a la validez convergente del instrumento, los resultados de los análisis de correlación bivariada evidencian asociaciones estadísticamente significativas directas y de magnitud media con: síntomas de depresión $(r=.39, p<.001)$, ansiedad $(r=.37, p<.001)$ y estrés psicológico $(r=.36, p<.001)$. Por otra parte, se evidencia una correlación estadísticamente significativa, inversa y muy débil con la intención de buscar ayuda psicológica $(r=-.17, p<.01)$.

Finalmente, la muestra general obtiene un puntaje promedio en la escala de autoocultamiento que se ubica entre el nivel 3 "Neutral" y 4 "De acuerdo" ( $M=3.16, D E=.84)$.

\section{Discusión}

El presente estudio tuvo por objetivo evaluar las propiedades psicométricas de la versión adaptada de la Escala de Auto-Ocultamiento (SCS) de Larson y Chastain (1990) en una muestra de universitarios chilenos. Para cumplir este objetivo se realizó en una primera instancia la traducción y adaptación de la escala original, posterior a esto se exploró la estructura factorial de la versión adaptada de la escala, se evaluó su invarianza entre hombres y mujeres universitarios, se determinó su confiabilidad y finalmente se exploró su validez convergente con las variables: sintomatología ansiosa y depresiva, estrés psicológico e intención de buscar de ayuda psicológica.

La versión final adaptada de la escala quedó compuesta por ocho ítems, exhibiendo una estructura unidimensional, que confirma la estructura del instrumento original y de los estudios posteriores hechos en población universitária.
Además, la escala muestra un nivel de consistencia interna adecuado $(\omega=.85)$.

La escala evidenció una invarianza escalar parcial, donde sólo los ítems 3 ("Si compartiera todos mis temas más íntimos con mis amigos/as, les agradaría menos") y 4 ("Hay muchas cosas sobre mí que me guardo") variaron entre hombres y mujeres. Ello podría indicar que lo medido por esos dos reactivos, tiene un significado diferente para los hombres y mujeres de la presente muestra, o bien que experimenten de forma diferente estos aspectos del auto-ocultamiento. Desde esta perspectiva, es relevante que futuros estudios indaguen estos aspectos, a través de técnicas que permitan pesquisar los significados y comprender cómo lo entiende cada grupo.

Con relación a la validez convergente, la escala convergió en la dirección esperada y de manera significativa con las medidas de depresión, ansiedad, estrés psicológico (DASS21) lo que resulta consistente con lo reportado en estudios de meta-análisis, donde se evidencia que el acto de auto-ocultar información personal y relevante a otros se vincula consistentemente con desenlaces negativos en salud mental, caracterizada por un aumento de la sintomatología ansiosa y depresiva, y mayores niveles de estrés (Larson et al., 2015; Mendoza et al., 2018). Además, se evidenció una correlación directa con la intención de buscar ayuda psicológica (GHSQ$\mathrm{V})$, que fue de magnitud débil. Esto es consistente con los coeficientes de correlación que se han reportado en estudios anteriores (ej. $r=-.14$; Kelly \& Achter, 1995), y podría vincularse a que esta relación pudiese estar mediada por las actitudes hacia la búsqueda de ayuda psicológica, tal como lo plantea el modelo de búsqueda de ayuda psicológica de Cramer (1999), cuya estructura relacional ha sido corroborada en estudios 
posteriores (Cepeda-Benito \& Short, 1998; Kelly \& Achter, 1995; Leech, 2007; Yang et al., 2016). De esta forma, es necesario que futuros estudios que aborden el impacto el auto-ocultamiento en conductas como la búsqueda de ayuda psicológica, midan las actitudes (y sus componentes).

La escala original de diez ítems mide tres aspectos del auto-ocultamiento en una única dimensión: (a) la tendencia a mantener las cosas para uno mismo; (b) la posesión de secretos angustiantes o pensamientos negativos sobre sí mismo, que han sido compartidos con pocas personas; y (c) la aprehensión de revelar información personal. Pese a que la versión resultante en este estudio consta de ocho ítems, al revisar su contenido logran cubrir los tres aspectos antes mencionados en términos generales, con la salvedad de que -en el segundo aspecto- los ítems abordan los pensamientos negativos y no los secretos angustiantes. Esto se debe a que la escala sufrió cambios a partir de la adaptación del lenguaje, específicamente respecto de la eliminación de la palabra "secreto" mencionada en el apartado de resultados. De esta manera, considerando las diversas etapas del estudio y la inclusión de una evaluación de validez de contenido (poco usual en investigaciones previas con la escala), el presente instrumento se hace cargo de la adaptación del lenguaje al contexto, resguardando la validez de su contenido. Sin embargo, podría beneficiarse -en estudios posteriores- de la incorporación de ítems que aborden el componente angustioso del segundo aspecto que declara medir la escala original, que dada su relevancia teórica - podría agregar aspectos importantes en la medición de un constructo complejo como el auto-ocultamiento.

En línea con lo mencionado anteriormente, diversos estudios confirman la estructura unidimensional de la escala (ej. Constantine et al., 2004; Cramer \& Barry, 1999; Larson et al., 2015), pese a que se abordan tres elementos teóricos relevantes al constructo. Esto podría deberse a la extensión del instrumento, en donde la inclusión de más ítems podría eventualmente alterar su estructura, dependiendo de cuán relacionados estén teóricamente entre sí.

Particularmente, la población en la cual se validó esta escala ha sido descrita en la literatura internacional como un grupo vulnerable a sufrir trastornos de salud mental, y que comparativamente presenta peores indicadores de salud mental que sus equivalentes etarios no universitarios (Aceijas et al., 2017). En el contexto chileno, esta tendencia parece replicarse, evidenciándose altas cifras de sintomatología depresiva, ansiedad y estrés psicológico en este grupo (Barrera-Herrera et al., 2019). Teniendo en cuenta que el auto-ocultamiento se relaciona con estos trastornos, resulta un aporte contar con una medida válida y confiable que permita estimar los niveles del auto-ocultamiento en una población en que es necesario comprender los problemas de salud mental.

En la presente muestra, el nivel de autoocultamiento alcanzado es medio (correspondiente a la categoría "Neutral"), lo que podría indicar que esta variable no se observa exacerbada en la muestra en estudio. Sin embargo, este resultado debe ser interpretado con cautela, dado que aún se requiere la aplicación de la escala validada a otras muestras de universitarios para tener mayor precisión sobre este aspecto y además examinar las diferencias que se evidenciaron en los ítems 3 y 4 en el análisis de invarianza.

Por otra parte, y pese a no ser un objetivo directo de este estudio, llama la atención que el porcentaje de estudiantes universitarios en actual tratamiento psicológico sea tan bajo, versus los altos niveles de sintomatología depresiva, ansiosa y de estrés psicológico evidenciados en la muestra. Si bien, la utilización de servicios psicológicos en esta población resulta problemática (Eisenberg, Hunt, Speer, \& Zivin, 2011), este resultado pone de manifiesto la relevancia de comprender mejor los factores que intervienen en este proceso. Una variable que podría explicar este resultado es la utilidad percibida del tratamiento psicológico anterior, en donde se observa que se percibe como una experiencia poco útil, lo cual podría frenar los intentos de consultar en la actualidad, pese a presentar sintomatología de salud mental.

Respecto de las características de este instrumento, al ser una escala breve, de fácil y rápida aplicación y auto-administrada, puede ser utilizada para identificar factores asociados a este constructo en población local y grupos en riesgo de sufrir desenlaces en salud desfavorables que han 
mostrado asociación con el auto-ocultamiento, como los mencionados anteriormente.

Este estudio tiene fortalezas y limitaciones. Es uno de los primeros estudios en Chile que aborda la adaptación y validación de la escala de autoocultamiento de Larson y Chastain (Larson \& Chastain, 1990); proceso riguroso realizado en etapas sucesivas, comenzando con la traducción y adaptación de la escala con el criterio de jueces expertos en la evaluación de su validez de contenido, para posteriormente pasar a una fase de evaluación de sus propiedades psicométricas donde se pudo confirmar el modelo teórico unidimensional, evaluar su invarianza entre hombres y mujeres, además de incluir evidencia de su confiabilidad y validez convergente.

Pese a que el proceso anteriormente descrito procuró ser exhaustivo, una de las limitaciones de este estudio es que no se incorporaron ítems que hayan emergido del presente grupo cultural. Futuras investigaciones que estén interesadas en la medición de este constructo podrían utilizar metodologías de abajo hacia arriba para la creación de ítems culturalmente pertinentes, que posteriormente puedan ser probados en la población local, de manera de complementar esta versión adaptada del instrumento.

En línea con lo mencionado anteriormente, sería interesante indagar si el constructo de autoocultamiento tiene alguna función cultural en el contexto local, dado que en otros contextos, por ejemplo, en grupos de latinos, se ha observado que el ocultar información personal negativa a la familia es una forma de mantener la armonía social (Interian et al., 2007). Sin embargo, no existe evidencia empírica que demuestre un escenario similar en el contexto local. No obstante, y aun cuando en este estudio no se realizó un examen cultural propiamente tal, cauteló la realización de un proceso de adaptación del lenguaje de los reactivos y las etapas sucesivas que se implementaron justificaron la eliminación de dos ítems, lo cual también contribuye a hacer esta escala más pertinente para la muestra. Asimismo, el análisis de invarianza aportó insumos respecto del significado de este constructo en hombres y mujeres universitarios del contexto local.

Finalmente, considerando las altas tasas de problemas de salud mental en la población universitaria, se hace necesario contar con instrumentos pertinentes a este segmento de la población (Del Valle et al., 2018), que estén adaptados al contexto y sean adecuados en términos psicométricos. De esta manera, no solo se contribuye a la comprensión teórica de esta temática, sino que en términos prácticos permite la pesquisa de casos que se constituyen en un grupo de riesgo para el desarrollo de patología física y psicológica, y que con menor probabilidad acudirían solos a buscar ayuda. De la misma manera, emerge el desafío para la disciplina de generar formas de intervención eficaces que promuevan la autoexpresión, y otros factores protectores de la salud mental en este segmento de la población.

\section{Agradecimientos}

Este trabajo fue financiado por Proyecto ANID Fondecyt de Iniciación \#11180115 y parcialmente financiado por ANID - Iniciativa Científica Milenio/Instituto Milenio para la Investigación en Depresión y PersonalidadMIDAP.

\section{Referencias}

Aceijas, C., Waldhäusl, S., Lambert, N., Cassar, S., \& Bello-Corassa, R. (2017). Determinants of health-related lifestyles among university students. Perspectives in Public Health. https://doi.org/10.1177/1757913916666875

Adimark. (2000). El Nivel Socioeconómico ESOMAR, Manual de aplicación. 1-8. Retrieved from http://www.microweb.cl/idm/documentos/ES OMAR.pdf

Akdoğan, R., \& Çimşir, E. (2019). Linking inferiority feelings to subjective happiness: Self-concealment and loneliness as serial mediators. Personality and Individual Differences, 149, 14-20. https://doi.org/10.1016/j.paid.2019.05.028

Almas, A. N., Grusec, J. E., \& Tackett, J. L. (2011). Children's disclosure and secrecy: Links to maternal parenting characteristics and children's coping skills. Social Development, 20(3), 624-643. https://doi.org/10.1111/j.1467- 
9507.2010.00602.x

Antúnez, Z., \& Vinet, E. V. (2012). Escalas de depresión, ansiedad y estrés (DASS - 21): Validación de la versión abreviada en estudiantes universitarios Chilenos. Terapia Psicologica, 30(3), 49-55. https://doi.org/10.4067/S071848082012000300005

Arnett, J. J. (2015). Emerging adulthood: The winding road from the late teens through the twenties (2nd ed; Oxford University Press, Ed.).

Auerbach, R. P., Mortier, P., Bruffaerts, R., Alonso, J., Benjet, C., Cuijpers, P., ... Kessler, R. C. (2018). WHO World Mental Health Surveys International College Student Project: Prevalence and distribution of mental disorders. Journal of Abnormal Psychology, 127(7), 623-638. https://doi.org/10.1037/abn0000362

Baader, T., Rojas, C., Molina, J. L., Gotelli, M., Alamo, C., Fierro, C., ... Dittus, P. (2014). Diagnóstico de la prevalencia de trastornos de la salud mental en estudiantes universitarios y los factores de riesgo emocionales asociados. Revista Chilena de Neuro-Psiquiatría, 52(3), 167-176.

https://doi.org/10.4067/S071792272014000300004

Barrera-Herrera, A., Neira-Cofré, M., RaipánGómez, P., Riquelme-Lobos, P., \& Escobar, B. (2019). Perceived social support and sociodemographic factors in relation to symptoms of anxiety, depression and stress in Chilean university students. Revista de Psicopatologia y Psicologia Clinica, 24(2), 105-115. https://doi.org/10.5944/rppc.23676

Castillo, L., \& Cano, M. (2007). Mexican American psychology: Theory and clinical application. En C. Negy, (Ed.), Cross-cultural psychotherapy: Toward a critical understanding of diverse client populations (pp. 85-102). Bent Tree Press, Inc.

Castillo, L. G., Perez, F. V., Castillo, R., \& Ghosheh, M. R. (2010). Construction and initial validation of the Marianismo Beliefs Scale. Counselling Psychology Quarterly, 23(2), 163-175.

https://doi.org/10.1080/09515071003776036

Cepeda-Benito, A., \& Short, P. (1998). Self- concealment, avoidance of psychological services, and perceived likelihood of seeking professional help. Journal of Counseling Psychology, 45(1), 58-64.

https://doi.org/10.1037/0022-0167.45.1.58

Constantine, M. G., Okazaki, S., \& Utsey, S. O. (2004). Self-concealment, social self-efficacy, acculturative stress, and depression in African, Asian, and Latin American International College Students. American Journal of Orthopsychiatry, 74(3), 230-241. https://doi.org/10.1037/0002-9432.74.3.230

Cramer, K. M. (1999). Psychological antecedents to help-seeking behavior: A reanalysis using path modeling structures. Journal of Counseling Psychology, 46(3), 381-387. https://doi.org/10.1037/0022-0167.46.3.381

Cramer, K. M., \& Barry, J. E. (1999). Psychometric properties and confirmatory factor analysis of the self-concealment scale. Personality and Individual Differences, 27(4), 629-637. https://doi.org/10.1016/S01918869(98)00222-0

Del Valle, M., Díaz, A., Pérez, M. V., \& Vergara, J. (2018). Confirmatory factor analysis of Perceived Self-Efficacy Scale in academic situations (EAPESA) in Chilean university students. Revista Iberoamericana de Diagnostico y Evaluación - e Avaliação Psicológica, 4(49), 97-106.

https://doi.org/10.21865/RIDEP49.4.08

Eisenberg, D., Hunt, J., Speer, N., \& Zivin, K. (2011). Mental health service utilization among College Students in the United States. The Journal of Nervous and Mental Disease, 199(5), 301-308. https://doi.org/10.1097/NMD.0b013e3182175 123

ELSOC-COES. (2018). Radiografía del cambio social: Análisis de Resultados Longitudinal Social de Chile ELSOC 2016-2018.

Friedlander, A., Nazem, S., Fiske, A., Nadorff, M. R., \& Smith, M. D. (2012). Self-concealment and suicidal behaviors. Suicide Life Threat Behavior, 42(3), 332-340.

https://doi.org/10.1016/j.physbeh.2017.03.040

Silva, A., Salinas-Oñate, N., Gómez-Pérez, D., \& Barrera-Herrera, A. (2022). Propiedades psicométricas del Cuestionario General de Búsqueda de Ayuda en universitarios del sur 
de Chile. Behavioral Psychology/Psicologia Conductual (artículo aceptado).

Gómez, A., Núñez, C., Agudelo, M., \& Grisales, A. (2020). Riesgo e ideación suicida y su relación con la impulsividad y la depresión en adolescentes escolares. Revista Iberoamericana de Diagnóstico y Evaluación - e Avaliação Psicológica, 54(1), 147-163. https://doi.org/10.21865/ridep54.1.12

Interian, A., Martinez, I. E., Guarnaccia, P. J., Vega, W. A., \& Escobar, J. I. (2007). A qualitative analysis of the perception of stigma among latinos receiving antidepressants. Psychiatric Services, 58(12), 1591- 1594. https://doi.org/10.1176/ps.2007.58.12.1591

Kahn, J. H., \& Garrison, A. M. (2009). Emotional self-disclosure and emotional avoidance: Relations with symptoms of depression and anxiety. Journal of Counseling Psychology, 56(4), 573-584.

https://doi.org/10.1037/a0016574

Kahn, J. H., \& Hessling, R. M. (2001). Measuring the tendency to conceal versus disclose psychological distress. Journal of Social and Clinical Psychology, 20(1), 41-65. https://doi.org/10.1521/jscp.20.1.41.22254

Kelly, A. E., \& Achter, J. A. (1995). Selfconcealment and attitudes toward counseling in University students. Journal of Counseling Psychology, 42(1), 40-46. https://doi.org/10.1037/0022-0167.42.1.40

Kelly, A. E., \& Yip, J. J. (2006). Is keeping a secret or being a secretive person linked to psychological symptoms? Journal of Personality, 74(5), 1349-1369. https://doi.org/10.1111/j.14676494.2006.00413.x

Larson, D. G., \& Chastain, R. L. (1990). Selfconcealment: Conceptualization, measurement, and health implications. Journal of Social and Clinical Psychology, 9(4), 439-455. https://doi.org/10.1521/jscp.1990.9.4.439

Larson, D. G., Chastain, R. L., Hoyt, W. T., \& Ayzenberg, R. (2015). Self-concealment: Integrative review and working model. Journal of Social and Clinical Psychology, 34(8), 705-774.

https://doi.org/10.1521/jscp.2015.34.8.705

Leech, N. L. (2007). Cramer's model of willingness to seek counseling: A structural equation model for counseling students. Journal of Psychology: Interdisciplinary and Applied, 141(4), 435-445.

https://doi.org/10.3200/JRLP.141.4.435-448

Masuda, A., Allen, G. E. K., Liu, C., \& Tully, E. C. (2021). The roles of self-concealment and perceived racial and ethnic discrimination in general psychological distress among racial and ethnic minority College students in the United States. International Journal for the Advancement of Counselling. https://doi.org/10.1007/s10447-021-09441-1

Masuda, A., Anderson, P. L., \& Edmonds, J. (2012). Help-seeking attitudes, mental health stigma, and self-concealment among African American College students. Journal of Black Studies, 43(7), 773-786. https://doi.org/10.1177/0021934712445806

Masuda, A., Anderson, P. L., Wendell, J. W., Chou, Y. Y., Price, M., \& Feinstein, A. B. (2011). Psychological flexibility mediates the relations between self-concealment and negative psychological outcomes. Personality and Individual Differences, 50(2), 243-247. https://doi.org/10.1016/j.paid.2010.09.037

Masuda, A., \& Boone, M. S. (2011). Mental health stigma, self-concealment, and helpseeking attitudes among Asian American and European American College students with no help-seeking experience. International Journal for the Advancement of Counselling, 33(4), 266-279. https://doi.org/10.1007/s10447-011-9129-1

Masuda, A., Boone, M. S., \& Timko, C. A. (2011). The role of psychological flexibility in the relationship between self-concealment and disordered eating symptoms. Eating Behaviors, 12(2), 131-135. https://doi.org/10.1016/j.eatbeh.2011.01.007

Masuda, A., Tully, E. C., Drake, C. E., Tarantino, N., Ames, A. M., \& Larson, D. G. (2017). Examining self-concealment within the framework of psychological inflexibility and mindfulness: A preliminary cross-sectional investigation. Current Psychology, 36(1), 184-191. https://doi.org/10.1007/s12144-015-9399-6

Masuda, A., Wendell, J. W., Chou, Y. Y., \& Feinstein, A. B. (2010). Relationships among 
self-concealment, mindfulness and negative psychological outcomes in Asian American and European American college students. International Journal for the Advancement of Counselling, 32(3), 165-177. https://doi.org/10.1007/s10447-010-9097-x

McDonald, R. P. (1999). Test theory: A unified treatment. (Mahwah: La).

Mendoza, H., Goodnight, B. L., Caporino, N. E., \& Masuda, A. (2018). Psychological distress among Latina/o College Students: The roles of self-concealment and psychological inflexibility. Current Psychology, 37(1), 172179. https://doi.org/10.1007/s12144-016-9500-9

Mendoza, H., Masuda, A., \& Swartout, K. M. (2015). Mental health stigma and selfconcealment as predictors of help-seeking attitudes among Latina/o College Students in the United States. International Journal for the Advancement of Counselling, 37(3), 207222. https://doi.org/10.1007/s10447-015-9237-4

Micin, S., \& Bagladi, V. (2011). Salud mental en estudiantes universitarios: Incidencia de psicopatología y antecedentes de conducta suicida en población que acude a un servicio de salud estudiantil. Terapia Psicologica. https://doi.org/10.4067/S071848082011000100006

Ortiz, M., \& Fernández-Pera, M. (2018). Modelo de ecuaciones estructurales: Una guía para ciencias médicas y ciencias de la salud. Terapia Psicológica, 36(1), 51-58.

Schulenberg, J. E., Sameroff, A. J., \& Cicchetti, D. (2004). The transition to adulthood as a critical juncture in the course of psychopathology and mental health. Development and Psychopathology, 16(4), 799-806. https://doi.org/10.1017/S0954579404040015

Vogel, D., \& Wester, S. (2003). To seek help or not to seek help: The risks of self-disclosure. Journal of Counseling Psychology, 50(3), 351-361. https://doi.org/10.1037/0022-0167.50.3.351

Yang, S., Hsu, W., Moi, S., Chen, H., Chang, Y., Rd, H. E., \& City, T. (2016). Development Research the effects of psychological factors on intention to seek counselling in international students In Taiwan.

Yukawa, S., Tokuda, H., \& Jun, S. (2007). Attachment style, self-concealment and interpersonal distance among Japanese undergraduates. Perceptual and Motor Skills, 104(3), 1255-1261. https://doi.org/10.2466/PMS.104.4.1255-1261 
Apéndice A. Versión validada de la escala de auto-ocultamiento

¿En qué medida estás de acuerdo o en desacuerdo con las siguientes afirmaciones? Por marca número que representa la respuesta apropiada.

$\mathrm{Al}$ responder, toma en consideración lo que haces la mayor parte del tiempo.

\begin{tabular}{|c|c|c|c|c|}
\hline Muy en desacuerdo & En desacuerdo & Neutral & De acuerdo & Muy de acuerdo \\
\hline (1) & (2) & (3) & (4) & (5) \\
\hline
\end{tabular}

1. Tengo un tema personal importante que no he compartido con nadie

2. Si compartiera todos mis temas más íntimos con mis amigos/as, les agradaría menos

3. Hay muchas cosas sobre mí que me guardo

4. Cuando me pasa algo malo, tiendo a guardarlo para mí.

5. Contar un asunto personal que me angustia es complicado para mí

6. Tengo un tema personal tan íntimo, que si alguien me preguntara al respecto evitaría contestarle

7. Algunos de mis asuntos personales son demasiado vergonzosos como para compartirlos

8. Los pensamientos negativos que tengo sobre mí mismo/a no los comparto con nadie

\begin{tabular}{l|l|l|l|l|}
1 & 2 & 3 & 4 & 5 \\
1 & 2 & 3 & 4 & 5 \\
1 & 2 & 3 & 4 & 5 \\
1 & 2 & 3 & 4 & 5 \\
1 & 2 & 3 & 4 & 5 \\
1 & 2 & 3 & 4 & 5 \\
1 & 2 & 3 & 4 & 5 \\
1 & 2 & 3 & 4 & 5 \\
\hline
\end{tabular}

